\title{
Entre la enfermedad y la muerte: «Eutanasia»
}

\author{
Between illness and death: "Euthanasia"
}

Ma del Carmen Dubón-Peniche ${ }^{1 *}$ y Luis E. Bustamante-Leija²

${ }^{1}$ Dirección Médica, Hospital General de México Dr. Eduardo Liceaga; ${ }^{2}$ Dirección General de Arbitraje, Comisión Nacional de Arbitraje Médico. Ciudad de México, México

\section{Resumen}

Los argumentos a favor y en contra de la eutanasia exponen un panorama complejo, que será necesario discutir y decidir en México. En este artículo se abordan algunos aspectos relevantes, como la determinación legal de la muerte, el campo de intervención de la bioética, la terminología relacionada con la eutanasia, sus clasificaciones, los instrumentos internacionales promotores de los derechos humanos, y reflexiones sobre la importancia de los cuidados paliativos y el derecho que tiene el paciente en situación terminal para actuar conforme a sus objetivos, expectativas y creencias, en el contexto de sus relaciones familiares y sociales.

Palabras clave: Cuidados paliativos. Eutanasia. Muerte. Suicidio asistido.

\section{Abstract}

The arguments for and against euthanasia present a complex picture that will need to be discussed and decided in Mexico. This article addresses some relevant aspects such as the legal determination of death, the field of bioethics intervention, terminology related to euthanasia, its classifications, international instruments promoting human rights, as well as reflections on the importance of palliative care and the patient's right to be in a terminal situation to act in accordance with their goals, expectations and beliefs, in the context of their family and social relationships.

Key words: Palliative care. Euthanasia. Death. Assisted suicide.

"La muerte es un castigo para algunos, para otros un regalo, y para muchos un favor."

Séneca

\section{Introducción}

Para tratar el tema de la eutanasia es inevitable hablar de la muerte; el término proviene del latín mors-mortis, que significa cesación. Desde el punto de vista biológico, consiste en el cese de las funciones orgánicas de un ser vivo, más allá de las cuales no es posible su revivificación. En el derecho, la muerte de la persona extingue su personalidad en las relaciones jurídicas, en tanto que en medicina legal el diagnóstico clínico de muerte se basa en la abolición definitiva, irreversible y permanente de las funciones vitales del organismo.

\section{Correspondencia:}

*María del Carmen Dubón-Peniche

Dr. Balmis, 148

Col. Doctores, Del. Cuauhtémoc

C.P. 06720, Ciudad de México, México

Fecha de recepción: 25-07-2018

Fecha de aceptación: 15-12-2018

E-mail: maria.dubon@salud.gob.mx

DOI: 10.24875/CIRU.18000626

0009-7411/@ 2018 Academia Mexicana de Cirugía. Publicado por Permanyer. Este es un artículo open access bajo la licencia CC BY-NC-ND (http://creativecommons.org/licenses/by-nc-nd/4.0/).
Cir Cir. 2020;88(4):519-525

Contents available at PubMed

www.cirugiaycirujanos.com 
En ese sentido, el primer problema que se presenta es el momento de la determinación legal de la muerte, sus causas y la forma de certificar la defunción, pues son asuntos no solo de orden público, sino también de Salubridad General de la República.

La Ley General de Salud, en su Título IV «Pérdida de la Vida», dispone como criterios para la determinación legal de la muerte los siguientes:

Artículo 343.- Para efectos de este Título, la pérdida de la vida ocurre cuando se presentan la muerte encefálica o el paro cardiaco irreversible.

La muerte encefálica se determina cuando se verifican los siguientes signos:

- Ausencia completa y permanente de conciencia;

- Ausencia permanente de respiración espontánea, $y$

- Ausencia de los reflejos de tallo cerebral, manifestado por arreflexia pupilar, ausencia de movimientos oculares en pruebas vestibulares y ausencia a estímulos nociceptivos.

Se deberá descartar que dichos signos sean producto de intoxicación aguda por narcóticos, sedantes, barbitúricos o sustancias neurotrópicas. Artículo 344.- Los signos clínicos de la muerte encefálica deberán corroborarse por cualquiera de las siguientes pruebas:

- Electroencefalograma que demuestre ausencia total de actividad eléctrica, corroborado por un médico especialista;

- Cualquier otro estudio de gabinete que demuestre en forma documental la ausencia permanente de flujo encefálico arterial.

La muerte es uno de los acontecimientos más trascendentes, ya que significa el final de un cuerpo vivo, y mientras que para algunas personas representa el fin de su existencia, para otras es el comienzo de la vida eterna ${ }^{2}$. Es un hecho inherente a todos los seres vivos, y de manera particular en las personas su abordaje es complejo y supone las circunstancias de tiempo, modo y lugar en que ocurre ${ }^{3}$. Una forma de catalogar la muerte es por su causa (enfermedad, accidente, suicidio, homicidio, etc.), lo cual determina las posibilidades de intervención médica.

En resumen, las personas mueren por diferentes causas, en diversas circunstancias y ámbitos culturales, con distintos niveles de conciencia y diferentes percepciones de lo que constituye una buena muerte.

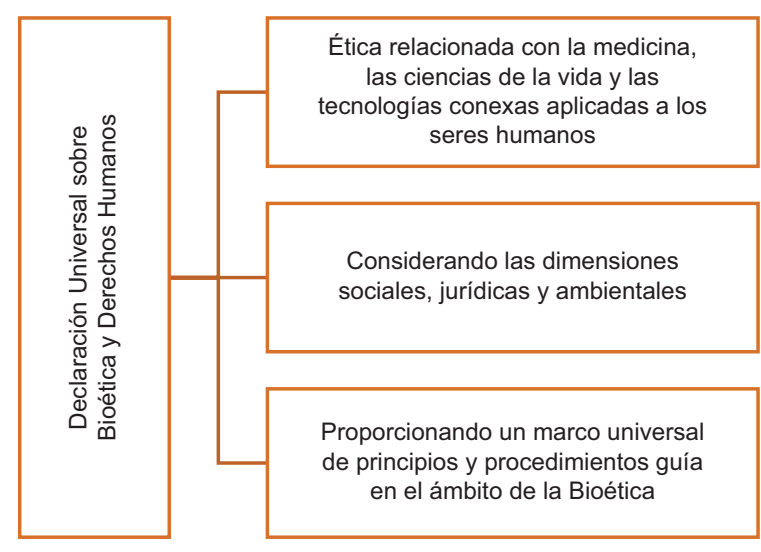

Figura 1. Declaración universal sobre bioética y derechos humanos (adaptada de: Organización de las Naciones Unidas para la Educación, la Ciencia y la Cultura ${ }^{5}$ ).

La relación que cada uno de nosotros establece con la muerte se teje en el plano más íntimo, a través de la conciencia personal, matizada por la época, las creencias y los conocimientos, y en base a ello cada cual vuelve la vista para buscar un sentido a su propia muerte ${ }^{4}$.

Los rápidos adelantos de la ciencia y sus aplicaciones tecnológicas en la medicina han suscitado distintos enfoques éticos relacionados con la eutanasia, mismos que ameritan ser explorados, considerando no solo el respeto a la dignidad de la persona, sino también el respeto universal y la observancia de los derechos humanos y las libertades fundamentales.

La vida es un derecho universal reconocido en constituciones políticas y normas de diferentes países, así como en instrumentos internacionales, entre los que destacan la Declaración Universal de los Derechos Humanos, la Declaración Universal sobre Bioética y Derechos Humanos, la Carta de Derechos Fundamentales de la Unión Europea y la Convención Americana de los Derechos Humanos. En este contexto, es evidente que el derecho internacional actúa como uno de los principales promotores de los derechos humanos (Fig. 1)

Existen diferentes perspectivas para situar el análisis de la eutanasia, el cual invariablemente debe reconocer sus consecuencias en el Derecho positivo. El campo de intervención de la bioética es amplio, pues sustenta su reflexión con la filosofía, la medicina, el derecho y la sociología, entre otras disciplinas. Dada la complejidad de su objeto, enlaza e integra distintos conocimientos a fin de responder ante diferentes argumentos y posturas que van surgiendo con 
los avances científicos y tecnológicos, los cuales en las ciencias médicas han representado la posibilidad de transformar el proceso salud-enfermedad en las personas, generando mayores expectativas en cuanto a la prolongación de la vida, y de manera especial en pacientes en estado grave, crítico o terminal.

En ese sentido, si bien es cierto que el desarrollo de la ciencia y la tecnología implica razonamientos éticos en sus aplicaciones, también lo es que no todo lo científicamente y técnicamente posible puede ser justificable desde un punto de vista ético y jurídico.

La bioética exige de la sociedad la necesidad de deliberar sobre los problemas relacionados con el medio ambiente, la vida y el cuerpo de las personas, así como la responsabilidad con las futuras generaciones, entre otros aspectos ${ }^{6}$.

Históricamente, la relación médico-paciente ha sido el elemento central de la ética médica, pues es a partir de esta relación que adquieren sentido los procesos de atención médica. En ese orden de ideas, es necesario reflexionar sobre un tema tan polémico al orientar la atención: saber advertir la línea de demarcación que permite distinguir lo permisible de lo no permisible ${ }^{7}$. En efecto, la decisión en cuanto a la indicación, la realización o la suspensión de procedimientos diagnóstico-terapéuticos en pacientes con enfermedad terminal no es fácil, y en este campo surge el apoyo de los Comités Intrahospitalarios de Bioética, los cuales entre sus objetivos contemplan asesorar al personal de salud y los usuarios en relación con los problemas y los dilemas bioéticos que surgen en la prestación de servicios de atención médica y docencia en salud, desde una perspectiva laica y científica ${ }^{8}$.

Lograr claridad conceptual resulta fundamental para tener la posibilidad de abordar los paradigmas morales que se presentan en el manejo de los pacientes y sus enfermedades por parte de los prestadores de servicios de atención médica, quienes diariamente enfrentan la toma de decisiones en las que entran en juego conflictos de valores.

\section{Eutanasia, concepto y términos relacionados}

La Organización Mundial de la Salud refiere que las definiciones de la eutanasia no son precisas y pueden variar de una persona a otra, pero con algunos elementos en común. La mayoría de los comentaristas limitan su descripción a la eutanasia directa o activa, que se puede dividir en tres categorías: 1) el homicidio intencional de quienes han expresado su deseo competente y libre; 2) el suicidio profesionalmente asistido; y 3) el homicidio intencional de los recién nacidos que tienen anomalías congénitas que pueden o no amenazar la vida, a menudo por retención de alimento. El término eutanasia pasiva es engañoso e inexacto; se refiere a prácticas en pacientes moribundos, como son el retiro del tratamiento que ha demostrado ser de ningún beneficio, o bien no iniciar un tratamiento que se considere de ningún beneficio, y la aplicación vigorosa de tratamiento para el dolor, aunque esto posiblemente pueda acortar la vida. Ya que estas decisiones se pueden hacer cerca del final de la vida, la muerte puede seguir pronto, pero no invariablemente, y se han etiquetado estas prácticas como eutanasia9.

Para la Asociación Médica Mundial, la eutanasia es considerada como la realización de forma intencional y con conocimiento de un acto con la clara intención de poner término a la vida de otra persona, siempre y cuando esta sea competente, se encuentre informada del estado incurable de su enfermedad y haya pedido voluntariamente terminar con su vida ${ }^{10}$.

La eutanasia está definida por la Real Academia Española como «Acción u omisión que, para evitar sufrimientos a los pacientes desahuciados, acelera su muerte con su consentimiento o sin él» y como "Muerte sin sufrimiento físico»"1.

Etimológicamente, "eutanasia» es una palabra que deriva de sus raíces griegas, eu (bueno) y thanatos (muerte), y significa buena muerte o bien morir. Lo contrario de la eutanasia es la distanasia (obstinación terapéutica o ensañamiento terapéutico), por sus raíces griegas dis (mal, algo mal hecho) y thanatos (muerte), y consiste en retrasar el acontecimiento de la muerte todo lo posible aunque no exista esperanza alguna de curación, sin tomar en cuenta la calidad de vida del enfermo; es decir, se trata de la prolongación de la agonía, el sufrimiento y la muerte de un paciente ${ }^{12}$. La obstinación terapéutica no es otra cosa más que la adopción de medidas desproporcionadas o inútiles con el objeto de alargar la vida en situación de agonía ${ }^{13}$.

La ortotanasia se refiere a permitir que la muerte ocurra en su tiempo cierto, "cuando deba ocurrir», y los profesionales de la salud están capacitados para otorgar al paciente todos los cuidados y tratamientos para disminuir el sufrimiento sin alterar el curso de la enfermedad ni, por ende, el curso de la muerte. Existe también la limitación del esfuerzo terapéutico, consistente en retirar o no iniciar medidas terapéuticas porque los profesionales de la salud estiman que en la 
situación concreta del paciente son inútiles, ya que solo conseguirían prolongarle la vida sin posibilidad de brindarle una recuperación funcional con una calidad de vida mínima. La omisión o el retiro de los medios extraordinarios también se ha denominado adistanasia $^{14}$.

Por su parte, el suicidio consiste en el acto mediante el cual una persona se priva voluntariamente de la vida, y el suicidio asistido se refiere a que un tercero proporcione algún medio efectivo al paciente con el fin de que este pueda darse muerte por sí mismo.

En cuanto a su clasificación, la eutanasia ha sido catalogada según su género, surgiendo así una variedad de categorías, entre las que destacan ${ }^{15-18}$ :

- Por su finalidad:

- Eugenésica: muerte a personas deformes o enfermas, para no degenerar la raza.

- Económica: eliminación de enfermos incurables, discapacitados o ancianos, para aligerar a la sociedad de personas inútiles que suponen elevados costos económicos y asistenciales.

- Piadosa: por sentimiento de compasión hacia la persona que está soportando graves sufrimientos, sin esperanza de sobrevivir.

- Solidaria: muerte indolora a personas desahuciadas con el fin de utilizar sus órganos o tejidos con fines terapéuticos.

- Por la modalidad de acción:

- Activa: solicitada por el propio paciente en etapa terminal y provocada por la acción positiva de un médico o personal de salud.

- Pasiva: muerte de un paciente por omisión de un tratamiento terapéutico necesario, interrupción de la terapia con la finalidad de no prolongar los sufrimientos de un enfermo al final de la vida.

Esta distinción entre eutanasia activa y pasiva tiende a desaparecer al sustituirse la terminología de «eutanasia pasiva» por la de «limitación del esfuerzo terapéutico", aceptándose de forma general que no se justifica prolongar el sufrimiento del enfermo de manera completamente inútil.

- Por la intención:

- Directa: provocación de la muerte inmediata y de manera deliberada con medios certeros.

- Indirecta o lenitiva: acción u omisión que indirectamente (por efecto secundario) provoca la muerte.

- Por el contenido volitivo:
- Voluntaria: se realiza a petición del paciente con su consentimiento informado, expreso y consciente.

- No voluntaria, que contempla dos argumentos:

- Muerte del paciente que no es capaz de entender la opción entre la vida y la muerte.

- Se impone a un paciente en contra de su voluntad, contraviniendo sus propios deseos.

La modalidad no voluntaria es elemento de debate al entenderse que se trata de una imposición, y por lo que tanto se hablaría de homicidio.

- Por la autoría:

- Heterónoma: interviene una o varias terceras personas.

- Autónoma: actúa el propio paciente. En esta modalidad se hablaría de suicidio.

Independientemente de las distintas clasificaciones, el propósito es el mismo: terminar con la vida de una persona.

\section{Medicina paliativa}

En la actualidad, los cuidados paliativos, incluyendo la psicología del moribundo y el duelo, ocupan un lugar preponderante; son un enfoque de mejora de la calidad de vida de pacientes y familias que se enfrentan a los problemas asociados con enfermedades que amenazan la vida, a través de la prevención y el alivio del sufrimiento por medio de la identificación temprana y la correcta evaluación y tratamiento del dolor, así como de otros problemas físicos, sociales y espirituales. Son especialmente importantes en países poco desarrollados, donde con elevada frecuencia se presentan casos diagnosticados en etapas avanzadas de la enfermedad, cuando ya no es posible la curación, pero se puede ayudar a disminuir el sufrimiento mediante intervenciones asequibles.

En nuestro país, el 5 de enero de 2009 se publicó en el Diario Oficial de la Federación el Decreto por medio del cual se reformó la Ley General de Salud efectuando adiciones en materia de cuidados paliativos. Dicha Ley dispone que toda persona mayor de edad, en pleno uso de sus facultades mentales, puede, en cualquier momento e independientemente de su estado de salud, expresar su voluntad por escrito, ante dos testigos, de recibir o no cualquier tratamiento, en caso de que llegase a padecer una enfermedad y estar en situación terminal y no le sea posible manifestar dicha voluntad. 


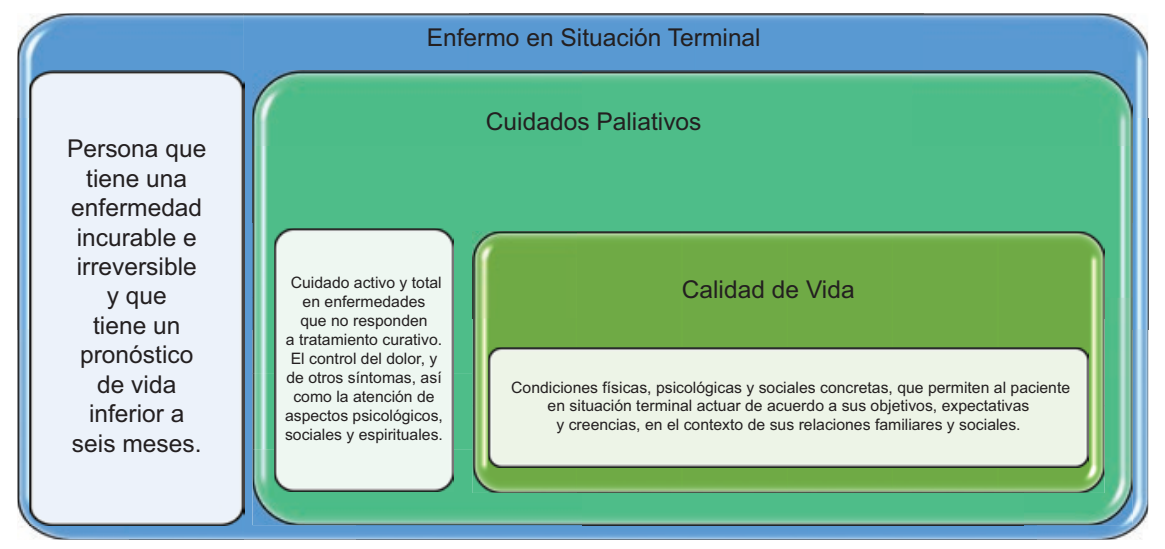

Figura 2. Enfermo en situación terminal (adaptada de: Norma Oficial Mexicana NOM-011-SSA3-201419).

Los cuidados paliativos consisten en el cuidado activo y total de aquellas enfermedades que no responden a un tratamiento curativo: el control del dolor y de otros síntomas, así como la atención de aspectos psicológicos, sociales y espirituales.

Un paciente susceptible de recibir cuidados paliativos es aquel que cursa con una enfermedad o situación avanzada, crónica, incurable, que puede incluir trastornos infecciosos, traumáticos, metabólicos, degenerativos u oncológicos, entre otros, con independencia del pronóstico del tiempo de sobrevida ${ }^{19}$. Sin embargo, los cuidados paliativos no deben reservarse solo para la última etapa de la vida, sino que deben aplicarse de manera conjunta al tratamiento que intenta modificar la enfermedad e incrementarse a medida que el paciente se acerca al final de la vida. Los cuidados paliativos también deben ser extensivos a la familia, incluso aún después de la muerte del paciente ${ }^{20}$.

Todos los pacientes sin expectativa curativa deben tener acceso a un nivel básico de cuidados paliativos. Se considera paciente terminal aquel que presenta una enfermedad avanzada, progresiva, incurable, con síntomas intensos, múltiples, multifactoriales, sin posibilidad de reaccionar positivamente a un tratamiento curativo, con amplias posibilidades de que le sobrevenga la muerte a causa de ese padecimiento. El paciente en situación terminal tiene derecho a recibir atención médica integral, así como a optar por recibir cuidados paliativos en su domicilio. En efecto, la autonomía es un principio fundamental de la bioética, y se refiere a la capacidad de realizar actos con conocimiento de causa, con información suficiente y sin coacción interna ni externa; es decir, la autonomía reconoce que la persona enferma tiene derecho a decidir libremente, después de haber recibido la información apropiada, entre las opciones clínicas disponibles, y reconoce también el derecho a negarse al tratamiento ${ }^{19}$.

El médico debe iniciar los cuidados paliativos en el momento en que se diagnostique el estado terminal y se informe al enfermo de las opciones, respetando su decisión y solicitando su consentimiento informado por escrito. Al respecto, el artículo 166 bis de la Ley General de Salud dispone lo siguiente ${ }^{21}$ :

I. Salvaguardar la dignidad de los enfermos en situación terminal, para garantizar una vida de calidad a través de los cuidados y atenciones médicas, necesarios para ello;

II. Garantizar una muerte natural en condiciones dignas a los enfermos en situación terminal;

III. Establecer y garantizar los derechos del enfermo en situación terminal en relación con su tratamiento;

IV. Dar a conocer los límites entre el tratamiento curativo y el paliativo;

$V$. Determinar los medios ordinarios y extraordinarios en los tratamientos; $y$

VI. Establecer los límites entre la defensa de la vida del enfermo en situación terminal y la obstinación terapéutica.

Cabe mencionar que la calidad de vida se refiere a las condiciones físicas, psicológicas y sociales concretas que permiten al paciente en situación terminal actuar de acuerdo con sus objetivos, expectativas y creencias, en el contexto de sus relaciones familiares y sociales $^{13}$ (Fig. 2).

Al hablar de cuidados paliativos, debe especificarse la distinción entre medidas ordinarias y extraordinarias. Las medidas ordinarias son las proporcionadas a las condiciones del enfermo, como el acompañamiento, la 
comunicación, la hidratación, la alimentación, los cambios de posición, el aseo y la curación de las heridas, así como los procedimientos y los medicamentos administrados para mitigar el dolor o suprimir síntomas molestos como náuseas, vómitos, mareos e insomnio, todo ello para disminuir el sufrimiento, con lo que se pretende que el paciente este más cómodo y tenga una mejor calidad de vida. Las medidas extraordinarias o desproporcionadas a las condiciones del enfermo no están indicadas en quienes, por lo avanzado de su enfermedad, complicaciones graves y falta de respuesta al tratamiento, el pronóstico es fatal a corto o mediano plazo; entre ellas se pueden mencionar el soporte respiratorio, los estudios diagnósticos invasivos, la cirugía, los antibióticos, la nutrición parenteral y las drogas vasoactivas, y lo mismo puede decirse de la quimioterapia y la radioterapia, que dan lugar a molestias y efectos indeseables. En este orden de ideas, es indudable que a los avances científicos y tecnológicos que han salvado muchas vidas y curado muchas enfermedades se ha sumado la medicina paliativa, la cual no es contraria a dichos avances, pero pugna por su adecuado uso, con un sentido humanista en el que prevalece el respeto a la voluntad y la autonomía del enfermo. El equipo de medicina paliativa interviene y acompaña al enfermo desde el momento inicial, durante toda la evolución de la enfermedad y el proceso diagnóstico y terapéutico, tiempo en que se fortalece la relación del médico con el paciente y la familia ${ }^{22}$.

\section{La eutanasia en distintos países}

La eutanasia ha sido manejada esencialmente por dos corrientes filosóficas fundamentadas en la ciencia y la religión, basándose en conocimientos y creencias socialmente desarrolladas, invocando la dignidad humana tanto para defenderla como para rechazarla. Para sus defensores, la dignidad humana del enfermo consiste en el derecho a elegir de manera libre el momento de su muerte. Para sus detractores, la dignidad humana es oponerse a este derecho por considerarlo una arbitrariedad frente a un asunto exclusivamente de orden divino, para algunos, o científico-legal para otros ${ }^{23}$.

Holanda fue el primer país en permitir la eutanasia con la aprobación de la Ley de comprobación de la terminación de la vida a petición propia y de auxilio al suicidio, en la cual se estipulan las condiciones del procedimiento que debe seguirse para que pueda llevarse a cabo ${ }^{24}$. Por su parte, Bélgica aprobó la Ley de muerte digna y eutanasia, la cual dispone que el paciente debe cumplir varios requisitos en su solicitud, ya que debe ser voluntaria, reflexionada y reiterada con certificación de varios médicos, quienes deben obtener la aprobación de una Comisión Federal Médica. Posteriormente, Luxemburgo aprobó la Ley sobre el derecho a una muerte digna.

En los Estados Unidos de Norteamérica, en Oregón es legal la eutanasia activa por la Ley de muerte con dignidad, la cual establece los requisitos para solicitar la medicación que termine una vida en forma humana y digna; se trata de una despenalización alejada de las legislaciones belga y holandesa.

En América Latina, Colombia es el único país donde se encuentra despenalizada la eutanasia para enfermos terminales que expresen su voluntad libre de poner fin a su vida, quedando los médicos que los asisten exentos de responsabilidad legal.

La eutanasia en México está penalizada. El Código Penal Federal contempla el delito de auxilio o inducción al suicidio, y aunque se considera como homicidio piadoso, pues la ayuda al enfermo terminal se brinda con la intención de aliviar su sufrimiento y a petición del propio enfermo, se persigue de oficio, y cuando se trata de un menor o enfermo mental, la pena se agrava y se considera homicidio calificado.

Artículo 312.- El que prestare auxilio o indujere a otro para que se suicide, será castigado con la pena de uno a cinco años de prisión; si se lo prestare hasta el punto de ejecutar él mismo la muerte, la prisión será de cuatro a doce años.

Artículo 313.- Si el occiso o suicida fuere menor de edad o padeciere alguna de las formas de enajenación mental, se aplicarán al homicida o instigador las sanciones señaladas al homicidio calificado o a las lesiones calificadas ${ }^{25}$.

El 7 de enero de 2008 fue promulgada la Ley de Voluntad Anticipada para el Distrito Federal (ahora Ciudad de México), con objeto de establecer y regular las normas, los requisitos y las formas de realización de la voluntad de cualquier persona con capacidad de ejercicio respecto a la negativa a someterse a medios, tratamientos o procedimientos médicos que pretendan prolongar de manera innecesaria su vida, protegiendo en todo momento la dignidad de la persona, cuando por razones médicas, fortuitas o de fuerza mayor sea imposible mantener su vida de manera natural. Las disposiciones establecidas en esta Ley son relativas a la práctica médica aplicada al enfermo en etapa terminal, consistente en el otorgamiento del tratamiento de los cuidados paliativos, protegiendo en todo momento su dignidad. 
Siguiendo a la Ciudad de México, otras entidades federativas cuentan con leyes que regulan la voluntad anticipada, entre ellas Aguascalientes, Chihuahua, Coahuila, Colima, Estado de México, Guanajuato, Guerrero, Hidalgo, Michoacán, Nayarit y San Luis Potosí.

Entre los argumentos de quienes se oponen a la eutanasia, uno de los más fuertes consiste en que legalizar la eutanasia llevaría de manera inevitable a abusos en el sentido de terminar con la vida de enfermos sin que ellos lo soliciten, ya sea por decisión del médico, de la familia o de ambos ${ }^{26}$.

También hay posturas que refieren que el derecho moral del paciente al respeto de su integridad y autonomía no es absoluto, y cuando ese derecho entra en conflicto con el de la integridad de otras personas surgen varias limitaciones. Una de ellas es el derecho del médico, pues el paciente no puede transgredir la integridad del médico como persona cuando se opone a la eutanasia por razones morales.

\section{Reflexiones finales}

Los argumentos a favor y en contra de la eutanasia exponen un panorama complejo, que será necesario discutir y decidir en México, y si bien la experiencia en otros países es de gran utilidad, se deben encontrar soluciones que respondan a las particularidades de nuestra cultura.

El tema es muy sensible y trascendente para suscitar un amplio debate, tanto en el plano ético como en el legal, que involucre no solo a la comunidad científica, sino que también exprese el sentir de la sociedad, tanto en el ámbito nacional como en el internacional, con argumentos fundamentales, referentes a la defensa de la dignidad humana y la preocupación para que el ejercicio de la medicina mantenga el sentido humanista que la ha caracterizado.

Dentro de su labor cotidiana, los prestadores de servicios de atención médica se enfrentan al fenómeno de la muerte, para lo cual deben ser capaces de realizar un análisis crítico y elegir la mejor opción para el enfermo y su familia, distinguiendo claramente entre prolongar la vida y prolongar la muerte.

Para lograr lo anterior, se considera indispensable que las instituciones de salud impulsen la formación bioética, así como la actualización médica continua en aspectos bioéticos y legales.

\section{Conflicto de intereses}

Los autores declaran que no existe conflicto de intereses.

\section{Bibliografía}

1. Ley General de Salud, Diario Oficial de la Federación de 27 de enero de 2017. (Consultado el 28 de junio de 2018.) Disponible en: http://www. diputados.gob.mx/LeyesBiblio/pdf/142_010616.pdf

2. López J, López O. Sobre la muerte: a quien pueda interesar. Rev Colomb Anestesiol. 2012:40:195-8.

3. Villamizar E. El morir y la muerte en la sociedad contemporánea. Gerencia y Políticas de Salud. 2002;1:66-80. (Consultado el 30 de junio de 2018.) Disponible en: http://revistas.javeriana.edu.co/index.php/gerepolsal/article/view/2902

4. Hernández F. El significado de la muerte. Revista Digital Universitaria, DGASCA-UNAM, México. 2006;7:1-7.

5. Organización de las Naciones Unidas para la Educación, la Ciencia y la Cultura. Declaración universal sobre bioética y derechos humanos. 33a sesión Conferencia General de la UNESCO, Francia, 2005. Consultado el 20 de junio de 2018. Disponible en: http://portal.unesco.org/ es/ev.php-URL_ID=31058\&URL_DO=DO_TOPIC\&URL_SECTION=201.htm

6. Gracia-Guillén D. De la bioética clínica a la bioética global: treinta años de evolución. Acta Bioethica, Universidad de Chile, Santiago, Chile. 2002;8:27-39.

7. Torralba F. Filosofía del cuidar. Mapfre Medicina, España. 2000;11:10110.

8. Comisión Nacional de Bioética. Guía nacional para la integración y el funcionamiento de los Comités Hospitalarios de Bioética. 5..$^{\text {a }}$ ed. México, 2015. (Consultado el 28 de junio de 2018.) Disponible en: http://www. mexico.salud.gob.mx/descargas/pdf/registrocomites/Guia_CHB_Final_ Paginada con forros.pdf.

9. Organización Mundial de la Salud. Technical paper: ethics of medicine and health. (Consultado el 28 de junio de 2018.) Disponible en: http:// applications.emro.who.int/docs/em rc42 7 en.pdf.

10. Asociación Médica Mundial. Declaración sobre la eutanasia. Resolución adoptada por la 39 Asamblea Médica Mundial, Madrid, España, reafirmada por la 200. a Sesión del Consejo de la AMM, Oslo, Noruega 2015. (Consultado el 28 de junio de 2018.) Disponible en: https:// www.wma.net/es/policies-post/resolucion-de-la-amm-sobre-la-eutanasia

11. Diccionario de la Real Academia Española. 2015. (Consultado el 28 de junio de 2018.) Disponible en: http://www.rae.es.

12. Cerdá G. Al filo de la muerte: cuidados paliativos vs eutanasia. En: Tomás Garrido GM, editor. Manual de bioética. Barcelona: Ariel Ciencia;2001. p. 445-61.

13. Norma Oficial Mexicana NOM-011-SSA3-2014. Criterios para la atención de enfermos en situación terminal a través de cuidados paliativos. Diario Oficial de la Federación, 9 de diciembre de 2014.

14. Félix Z, Geraldo S, Marques A, Garrido C, Costa M, Medeiros F. La eutanasia, distanasia y ortotanasia: una revisión integradora de la literatura. Ciência \& Saúde Coletiva. 2013;18:2733-46.

15. Azzolini A. Intervención en la eutanasia: ¿participación criminal o colaboración humanitaria? En: Eutanasia: aspectos jurídicos, filosóficos, médicos y religiosos. Instituto de Investigaciones Jurídicas, UNAM, México; 2001. p. 284-9

16. Matozzo L. ¿Buen morir... Buen matar? Buen control. En: El Derecho. No. 9071, 27 de agosto de 1996, y Revista Entre Círculos, del CM Salud, Círculos Médicos de San Isidro Vicente López, año I, Eros. 1, 2 y 3; 1998.

17. Marcos A. La eutanasia. Estudio filosófico-jurídico. Madrid: Pons Ediciones Jurídicas y Sociales; 2000. p. 1-358.

18. Trejo E. Legislación internacional y estudio de derecho comparado de la eutanasia. Dirección de Servicios de Investigación y Análisis. México: Cámara de Diputados; 2007. p. 25

19. Guía de Práctica Clínica en Cuidados Paliativos. México: Secretaría de Salud, 2010. (Consultado el 20 de junio de 2018). Disponible en: http:// www.cenetec.salud.gob.mx/descargas/gpc/CatalogoMaestro/445_GPC_ Cuidados_paliativos/GER_Cuidados_Paliativosx1x.pdf

20. Al-Mahrezi A, Al-Mandhari Z. Palliative care: time for action. Oman Medical Journal. 2016;31:161-3.

21. Norma Oficial Mexicana NOM-011-SSA3-2014. Criterios para la atención de enfermos en situación terminal a través de cuidados paliativos. México: Secretaría de Salud; 2010.

22. Gutiérrez-Sampeiro C, Ruiz-Canizales R, Arellano-Rodríguez S, Romero Zepeda H, Hall RT, García Camino B. Medicina paliativa en cirugía. Cir Cir. 2017:85:186-91.

23. Álvarez A. Algunos elementos para discutir la eutanasia. Rev Fac Med UNAM. 2007;50(1):28-30.

24. Código Penal Federal. Diario Oficial de la Federación, 20 de agosto de 2009. (Consultado el 30 de junio de 2018.) Disponible en: https://www. juridicas.unam.mx/legislacion/ordenamiento/codigo-penal-federal.

25. Acosta S. ¿Quién debe decidir? Rev Avances Médicos Cuba. 1996;3:60.

26. Álvarez A. Eutanasia y suicidio médicamente asistido. Rev Invest Clin. 2014;66:282-7. 